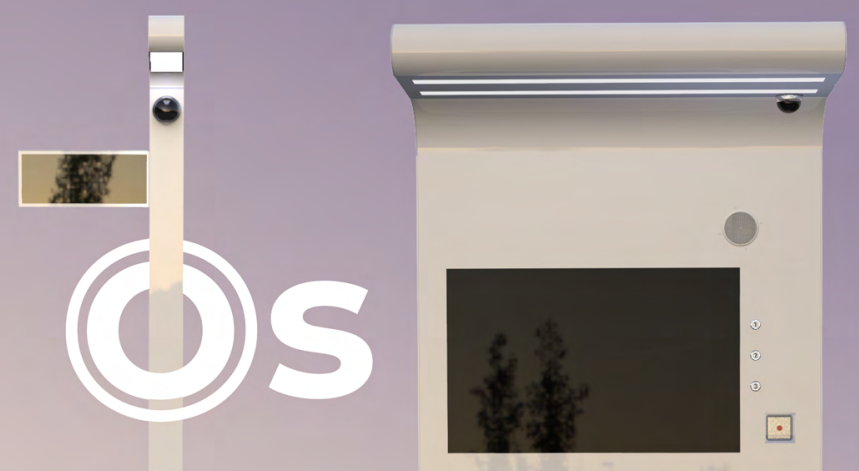

\title{
Diseño de sistema seguro de objetos inteligentes para el trayecto y espera de autobuses en Costa Rica
}

Design of a secure system of smart objects for the journey and wait of buses in Costa Rica

Nicole De-Vries-Gamboa ${ }^{1}$, Valeria Jiménez-Quirós ${ }^{2}$, Diana Vega-Vega ${ }^{3}$

N. De-Vries-Gamboa, V. Jiménez-Quirós, D. Vega-Vega, “Diseño de sistema seguro de objetos inteligentes para el trayecto y espera de buses en Costa Rica", IDI+, vol. 4, no 1, Jul., pp. 22-35, 2021.

doi) https://doi.org/10.18845/ridip.v4i1.5774

Fecha de recepción: 18 de diciembre de 2020

Fecha de aprobación: 1 de febrero de 2021

1. Nicole De-Vries-Gamboa Estudiante de Ingeniería en Diseño Industrial Instituto Tecnológico de Costa Rica Cartago, Costa Rica nicole.devriesgamboa@gmail.com (D) 0000-0003-2694-0896
2. Valeria Jiménez-Quirós Estudiante de Ingeniería en Diseño Industrial Instituto Tecnológico de Costa Rica Cartago, Costa Rica valejq21@gmail.com (D) 0000-0002-5422-8487

3. Diana Vega-Vega Estudiante de Ingeniería en Diseño Industrial Instituto Tecnológico de Costa Rica

Cartago, Costa Rica dsvegavega@gmail.com

(D) 0000-0002-5434-447X 


\section{Resumen}

Según la investigación realizada en este proyecto, se demuestran muchas deficiencias en el servicio del transporte público en Costa Rica, entre ellas, la percepción de inseguridad, situación que deja una amplia posibilidad para diseñar objetos inteligentes que ayuden a solventar esta problemática. Después de realizar pruebas que determinan las necesidades y requisitos más relevantes, se plantea la creación de OS bus stop y OS bus sign. Ambos objetos brindan seguridad al contar con iluminación y una cámara. El bus stop permite acceder a información concerniente al servicio y posee un botón de emergencia. Mientras que el bus sign (ubicado en el trayecto) muestra la dirección en la que se encuentran las distintas OS bus stops.

Además, se agrega un GPS a los autobuses, para que, desde los bus stops, se pueda acceder a su ubicación y, por ende, tiempo de espera. El OS bus stop cuenta con un asistente inteligente en la pantalla con el fin de proporcionar una guía para quienes no sepan cómo utilizar el sistema, lo que permite un aprendizaje más rápido de la interfaz $\mathrm{y}$, en caso de duda, obtener respuestas durante el uso.

Para este proyecto, se completan seis etapas que corresponden a las fases fundamentales del proceso proyectual. Se plantea el concepto, el diseño y la forma de solucionar su desarrollo como producto, además, se realiza una validación virtual, por medio de la simulación de diseño presentada en un vídeo. Esto para comprobar los aspectos de todas las etapas trabajadas y llegar a una síntesis del sistema.

\section{Palabras Clave}

Objeto inteligente; parada de autobus; seguridad; diseño; transporte público

\section{Abstract}

According to the research carried out, it was proved the existence of many deficiencies in the public transport service in Costa Rica, such as the perception of insecurity, which left a great possibility to design smart objects that help solving this problem. After carrying out tests to determine the most relevant needs and requirements, the creation of OS bus stop and OS bus sign was proposed. Both objects provide security by having lighting and a camera. The bus stop allows access to information concerning the service and has an emergency button. While the bus sign (located on the route) shows the direction in which the different OS bus stops are located.

Also, a GPS was added to the buses, so their location, and waiting time can be accessed from the bus stops. The OS bus stop has an intelligent assistant on the screen to provide a guide for those who don't know how to use the system, allowing the users to learn the use of the interface faster, and in case of any doubt they can get answers during the process. 
For this project, six stages were completed, corresponding to the fundamental phases of the project process. Once the concept, the design and the process to solve its development as a product had been raised, a virtual validation of the product was carried out, through the design simulation presented in a video. This, in order to verify the aspects of the stages worked and get a synthesis of the system.

\section{Keywords}

Smart object; bus stop; security; design; public transport

\section{Introducción}

El uso del transporte público es una actividad común para muchas personas a diario, desafortunadamente, en este servicio existen muchas deficiencias que representan una desventaja para la seguridad de estos ciudadanos. Dicha problemática se encuentra presente tanto en el trayecto hacia la parada como en la espera en ese lugar.

Solo en la provincia de San José, se efectúan más de 2 millones de viajes de transporte público diariamente [1], además, esta zona cuenta con muchos de los espacios más inseguros del país [2]. Donde se reportan casos de asaltos, acoso físico y verbal, agresiones y otros problemas que llegan a poner en riesgo a quienes utilizan este servicio. Asimismo, muchas de las paradas presentan problemas de acondicionamiento, algunas no se encuentran iluminadas, están mal colocadas y la mayoría no cuenta con ningún tipo de seguridad [3]. Aunado a ello, la información inmediata que se brinda sobre los autobuses es reducida [4] y su disponibilidad es limitada.

Debido a lo anterior, se planteó como objetivo el diseño de un sistema de objetos inteligentes, esto hace referencia a productos de uso cotidiano que interactúan con otros productos, entornos, personas y sistemas de tecnologías de información en formas totalmente nuevas. Perciben mediante sensores una situación en un contexto-entorno y son capaces de interpretar dicha información basándose en algoritmos para actuar en consecuencia con cierto nivel de autonomía y, de esta forma, mejorar la experiencia del usuario [5]. Además, que brinden seguridad durante el trayecto hacia la parada y en la espera del bus. Se propuso un sistema compuesto por dos objetos inteligentes, pues es la forma de brindar una mejor solución, basada en el contexto real, para resolver en mayor medida la problemática presentada, que no solo contempla la espera del bus, sino también el trayecto hacia la parada. Este abordaje surge a partir de preocupaciones externadas por usuarios reales del servicio de transporte público. Con la implementación de estos sistemas, se mejoraría la seguridad de la ciudad, un pilar fundamental de las ciudades inteligentes ${ }^{1}$.

1 Ciudades inteligentes: aquellas que disponen de un sistema de innovación y de trabajo en red para dotar a las ciudades de un modelo de mejora de la eficiencia económica y política permitiendo el desarrollo social, cultural y urbano. 


\section{Metodología}

Para el diseño de los objetos se desarrollaron cinco etapas que incluyen métodos pertenecientes al proceso proyectual. Estas etapas y sus métodos se explican a continuación:

\section{Conceptualizando la idea}

Para iniciar, se estudió el contexto de la situación actual, quiénes son los usuarios del servicio y qué problemas se presentan en las paradas. Se determinó, por medio de investigación de fuentes secundarias, que quienes más utilizarían los objetos serían personas jóvenes [6] y que las mujeres son quienes perciben más inseguridad en el transporte público [7]. Para su comprobación, se aplicó una encuesta respecto al uso de este transporte, donde se consultó: género, rango de edad, frecuencia de uso, motivo de uso, sensación de inseguridad, motivos de esta, así como el conocimiento y el acceso a la información relacionada al servicio de buses.

Se llevó a cabo una observación de usuarios, respecto a su desarrollo e interacción al momento de ir hacia una parada de autobús y cuando ya se encuentran ahí. Además, se efectuaron entrevistas a seis personas, entre los 18 y 24 años, por medios digitales, para documentar necesidades y hallazgos útiles a nivel de diseño. Estos hallazgos se colocaron en un árbol de problemas y fueron sintetizados mediante una tabla de necesidades y requerimientos.

Seguidamente, se definió el tamaño del mercado, utilizando datos obtenidos del Instituto Nacional de Estadística y Censos de Costa Rica del 2020, para determinar la cantidad de hombres y mujeres entre las edades definidas, y luego se obtuvo el porcentaje de personas que utilizan regularmente el transporte público [8]. Más adelante, se analizó lo existente donde se estudiaron factores como la seguridad brindada, la información que presentan, la infraestructura y se establecieron aspectos positivos y negativos para tomar en cuenta en el diseño. Por último, se validaron los requisitos por medio de una encuesta.

\section{Definiendo la funcionalidad}

En la segunda etapa, se hizo un diagrama de funciones basado en el árbol de funciones previamente definido, en este se especificó cuáles debería cumplir cada uno de los objetos. Se realizó una matriz morfológica con el fin de proponer distintas opciones para solucionar los requisitos planteados. Una vez definido lo anterior, se efectuó un análisis tecnológico para determinar en específico cuáles serían los componentes por utilizar.

Además, se propusieron distintos materiales para la construcción de los objetos y se establecieron formas de interacción entre las interfaces y los usuarios; luego, se concretó el funcionamiento del producto en el contexto de uso mediante diagramas de flujo. 
Posteriormente, para definir la arquitectura de los objetos, se desarrolló un diagrama de sistemas contemplando sus subsistemas, componentes y partes. Y, por último, se realizaron las primeras propuestas volumétricas por medio de bocetos a mano alzada explicando las funciones y la interacción, además, estas se validaron por medio de entrevistas con seis usuarios.

\section{Definiendo la forma}

Para la tercera etapa, se inició por definir el concepto de diseño, la descripción correspondiente, las variables consideradas, responder los $5 \mathrm{WH}$ (who, what, when, where, why, how), los objetivos y alcances del proyecto. Seguidamente, se realizó un análisis ergonómico, en el que se estudiaron percentiles a partir de tablas antropométricas con información recolectada en la Escuela de Diseño Industrial por el profesor Luis Carlos Araya, con el fin de determinar las medidas para la ubicación y tamaño de algunos componentes. Se determinaron los tiempos mínimos para las funciones inteligentes, además, factores por tomar en cuenta para la inclusión de poblaciones especiales y los determinantes de la interfaz. Por último, en este análisis se realizó una jerarquía de tareas, para generar un análisis de predicción del error humano y asegurarse de que fuera mínimo y solucionable.

Luego, en el análisis perceptual, se generó un vocabulario visual, que se utilizó dentro de los ejes semánticos, donde se eligió la perceptualidad que debían seguir los objetos. Más adelante, se realizó el diseño de múltiples alternativas y el datum ${ }^{2}$ tomando en cuenta los requisitos planteados en la etapa anterior para seleccionar la propuesta más adecuada y empezar a desarrollarla.

\section{Detallando la manufactura}

Para la cuarta etapa, se efectuó el cuaderno técnico de la propuesta seleccionada. Se explicó con detalle todos los aspectos técnicos y los procesos de manufactura correspondientes. Se presentan las funciones inteligentes, las interrelaciones entre estas y sus interfaces. Todas las partes correspondientes a los objetos se modelan en 3D y se muestran por medio de explosos. Se detallaron los materiales por utilizar en cada parte, además, se desarrollaron manuales, tanto para el uso como para la instalación, asimismo, se realizaron planos técnicos de las partes.

Para determinar los componentes estandarizados por utilizar, la especificación del proceso de manufactura y los ensambles tanto para el bus stop como para el bus sign, se consultó con expertos del campo. Por último, se calcularon los costos generales para la elaboración de un prototipo, esto contemplando el valor de los componentes y materiales respectivos a cada objeto.

2 Datum: técnica que permite evaluar alternativas de diseño usando criterios de selección. Se evalúa cada criterio para determinar si las propuestas de diseño son menos efectivas, igual o mejor que el producto seleccionado como "datum". 


\section{Simulación de diseño}

En la quinta y última etapa, se validaron los objetos, a causa de la situación actual de pandemia y la virtualidad adoptada por los cursos, se imposibilita construir una maqueta física, por lo que la comprobación del diseño se efectuó virtualmente. Se generó un vídeo con el fin de presentar el contexto de uso y entorno que tendrían los objetos, además, los usuarios que los utilizarían. Se muestra el concepto de diseño a partir del que fueron desarrollados, se mencionan las funciones que brindan los sistemas; también la interacción requerida entre objetos y usuario para cumplir algunas de las funciones. Por último, también se muestran los sistemas y subsistemas presentes en los objetos y los materiales e insumos que se propone utilizar para la creación de la propuesta de diseño.

\section{Resultados}

Al final de cada etapa, se obtuvieron hallazgos importantes que permitieron avanzar en el desarrollo del diseño. En la etapa conceptualizando la idea, se realizaron encuestas para definir el proyecto, en una de estas, se obtiene que el 100\% (44 personas) de los encuestados utiliza el servicio de transporte público y se han sentido inseguros utilizándolo, por lo que se les preguntó qué aspectos les generan la inseguridad (ver figura 1).

¿Qué aspectos le han generado inseguridad?

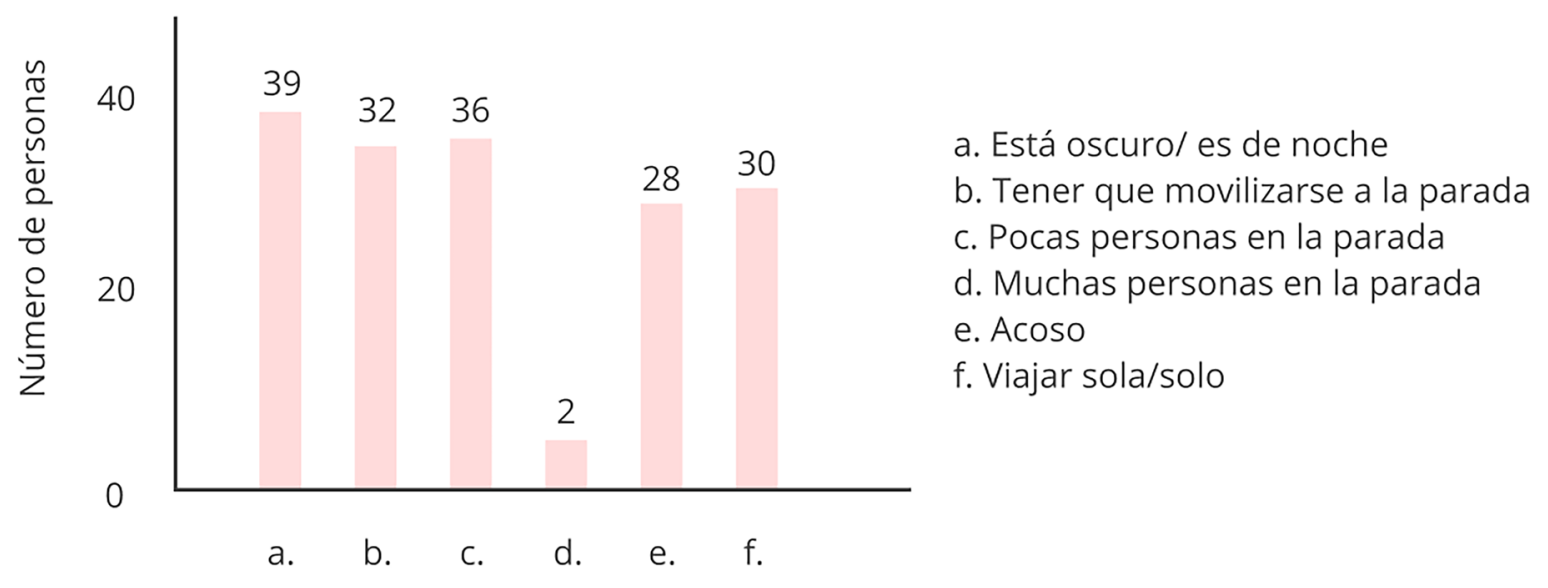

Aspectos generan inseguridad

Figura 1. Resultados encuesta: Transporte público, pregunta 7.

Fuente: Elaboración propia, 2020. 
Además, se determinaron las necesidades y requerimientos que se debían satisfacer, y se categorizaron entre deseables e indispensables (ver figura 2).

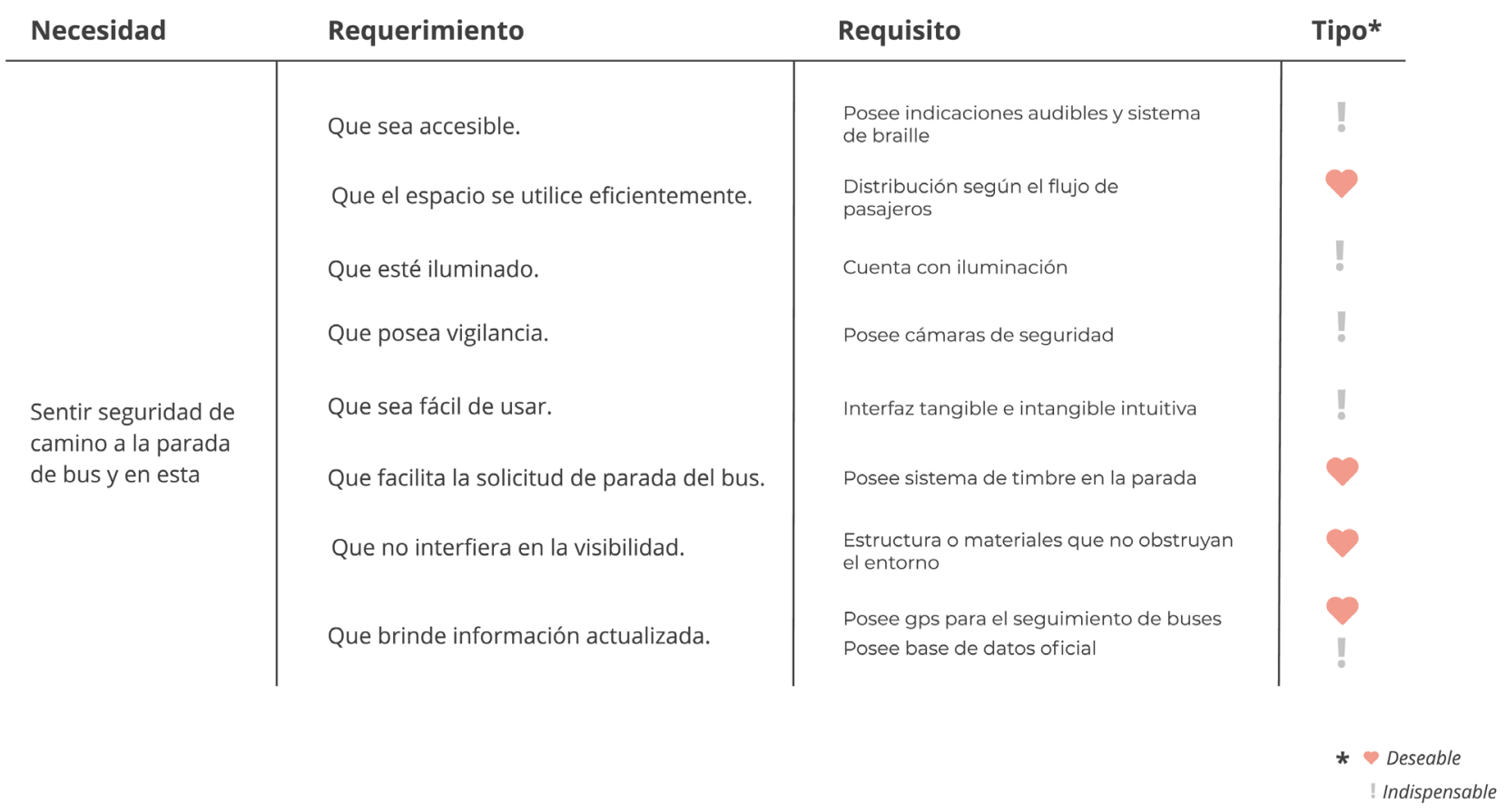

Figura 2. Tabla de necesidades y requerimientos.

Fuente: Elaboración propia, 2020.

Una vez validados los requisitos con usuarios reales, se determinó que todos se debían mantener, pues mejorarían la experiencia de los usuarios en el uso del sistema de buses disminuyendo la sensación de inseguridad. Además, se realizó un análisis de lo existente que reafirmó la necesidad de colocar objetos análogos, paradas de buses y "postes" informativos.

En la etapa definiendo la funcionalidad, primeramente, se establecieron las funciones que cumplirá cada producto, mediante un diagrama de funciones (ver figura 3). Se determinó que el sistema para espera de autobús tiene más funciones, ya que requería interacción con el usuario, mientras que el sistema de guía solo proporciona iluminación, muestra datos y posee un sistema de grabación (cámara). 


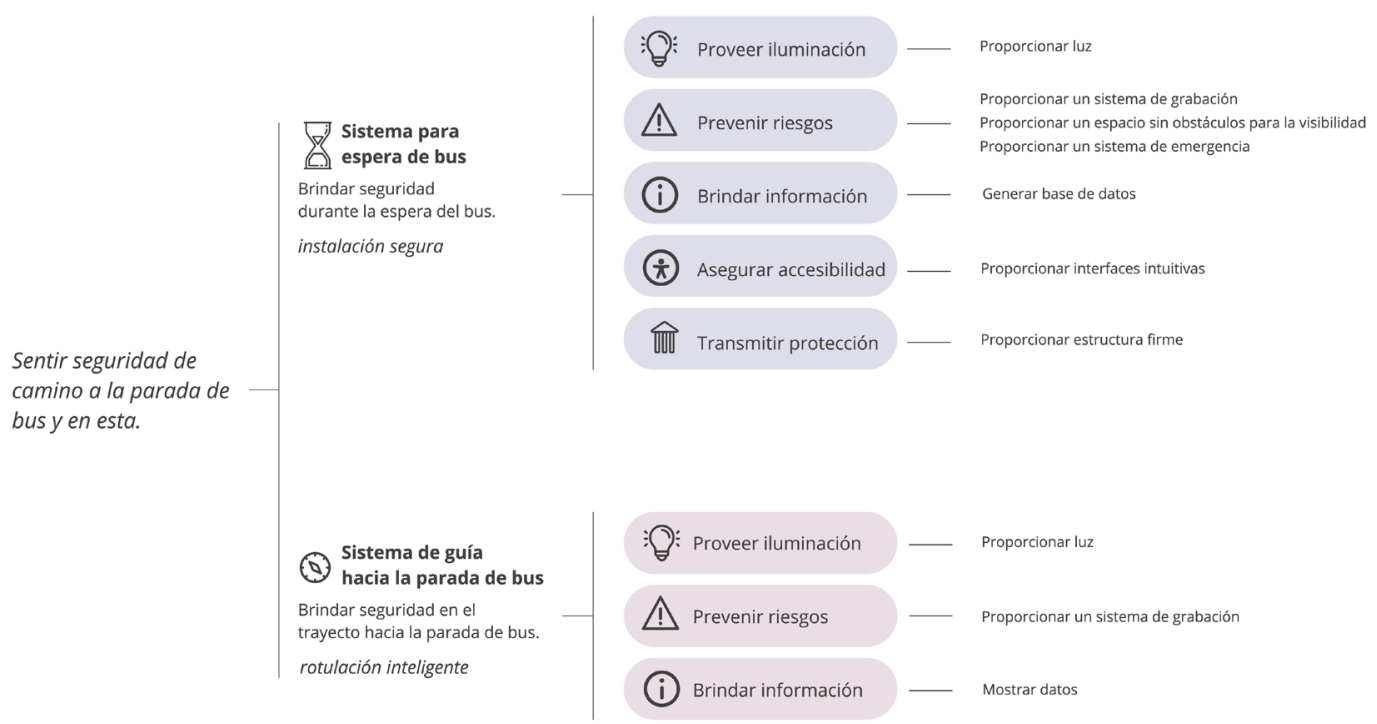

Figura 3. Diagrama de funciones

Fuente: Elaboración propia, 2020.

A partir de la información recopilada en la matriz morfológica, el análisis tecnológico, la propuesta de materiales y de interacción, los diagramas de flujo y el diagrama de sistemas (ver figura 4), se realizó la validación de los primeros bocetos con seis usuarios, lo que permitió analizar las características tanto positivas como negativas que se debían tomar en cuenta para continuar con el diseño.

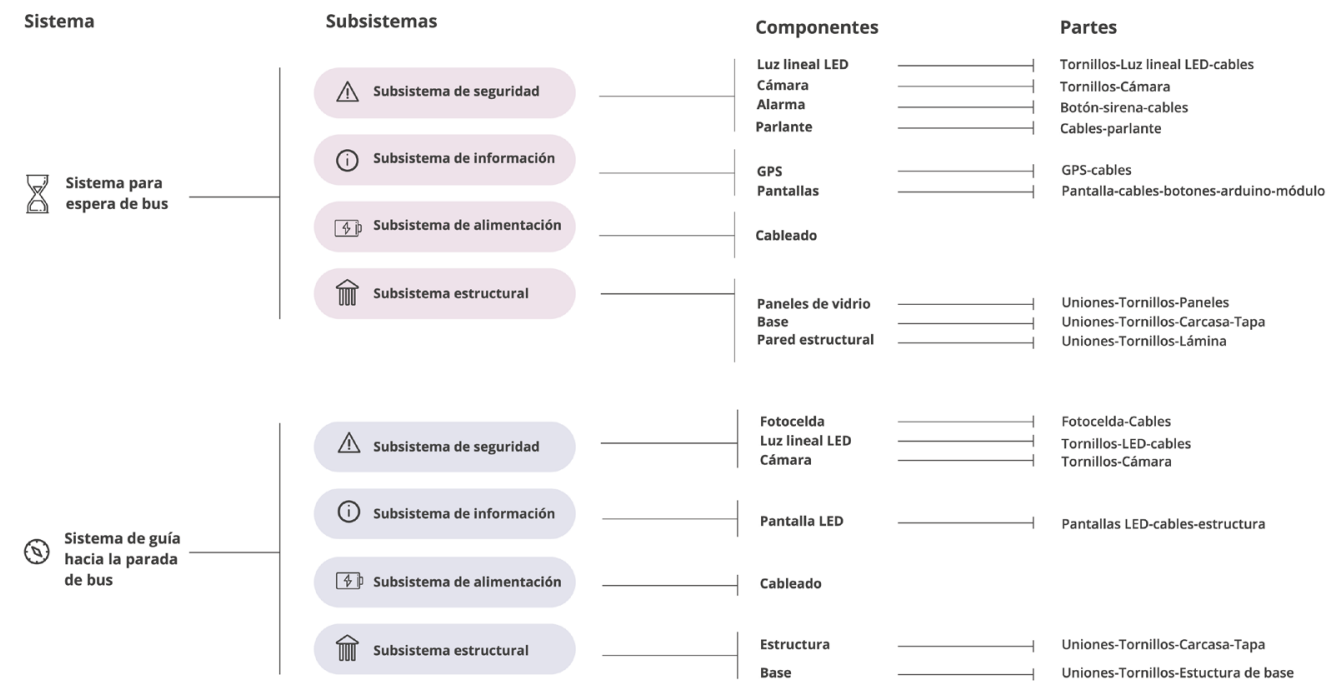

Figura 4. Diagrama de sistemas

Fuente: Elaboración propia, 2020. 
En la etapa de definición de la forma, se definió el concepto, con el objetivo de diseñar un sistema de objetos inteligentes que brindan seguridad durante el trayecto hacia la parada y en la espera del bus, por lo que se eligió el concepto de seguridad eficiente, con este se buscó generar una sensación de confianza a partir de sistemas diligentes ${ }^{3}$ que funcionan en conjunto para este fin. Y se determinaron los alcances del proyecto:

- Plantear un proceso de manufactura eficiente para el desarrollo de los sistemas.

- Utilizar software de modelado 3D para la representación gráfica de los sistemas.

- Desarrollar una propuesta de diseño que funcione como herramienta contra la inseguridad ciudadana.

A partir del vocabulario visual y el eje semántico (ver figura 5), se determinaron las características principales para que el producto se perciba más amigable y seguro:

- Bordes redondeados y suaves.

- Materiales metálicos.

- Uso de pantallas inteligentes.

- Botones con relieve y uso de luces para que sean intuitivos.

- Uso de una cromática de colores neutros (blanco, negro, grises).

- Acabados con brillo.

- Presencia de curvas en la morfología.

- Continuidad en los objetos y estructuras.

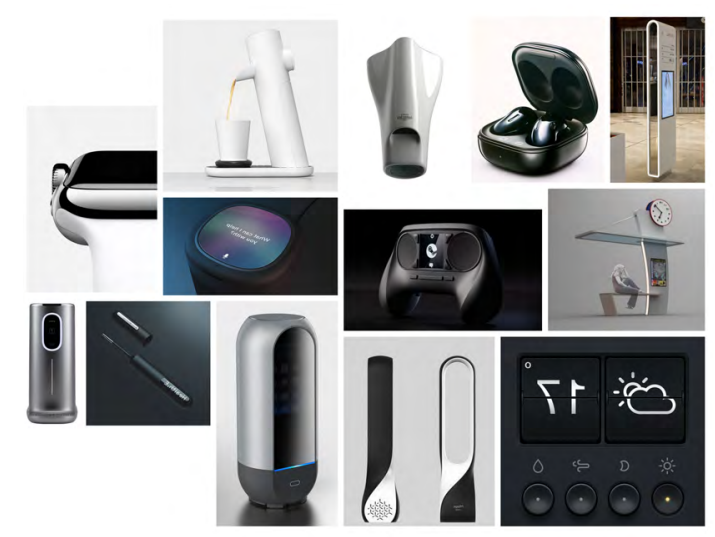

Figura 5. Eje semántico curvo-moderno

Fuente: Elaboración propia, 2020.

3 Diligente: que pone mucho interés, esmero, rapidez y eficacia en la realización de un trabajo o en el cumplimiento de una obligación o encargo. [9] 


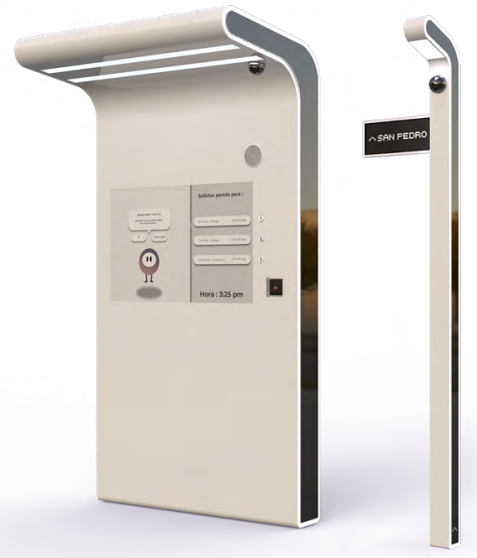

Figura 6. Render del OS bus stop y OS bus sign

Fuente: Elaboración propia, 2020.

Para la etapa de detallado de la manufactura, se realizó el modelado y render final de los objetos (ver figura 6), se especificaron las interfaces intangibles y también los materiales; para los materiales se utilizaron distintos criterios. Para el material de la estructura, la tapa y los laterales, se eligieron láminas de aluminio $6061 \mathrm{C}$ de $3 \mathrm{~mm}$ de espesor, ya que:

- $\quad$ Se consigue a un bajo costo.

- Cuenta con facilidad de limpieza.

- $\quad$ Tiene una larga vida útil.

- Gran resistencia.

- Material con gran maleabilidad.

Para el material de la base, se determinó el uso de láminas de acero inoxidable de $3 \mathrm{~mm}$ de espesor, ya que cuentan con:

- Mayor resistencia y durabilidad.

- $\quad$ Son menos deformables.

- $\quad$ Soportan mayores pesos y temperaturas.

Y, por último, para los laterales se eligió el vidrio templado FUERTEX, debido a que presenta:

- Gran resistencia a los impactos.

- $\quad$ En caso de que esta sufra un rompimiento, se fragmenta en pequeñas piezas.

- Cinco veces más fuerte que el vidrio flotado normal. 
Para el acabado, se propuso utilizar pintura por deposición electrostática y polimerizado 4 , luego, para la unión entre las piezas laterales de vidrio templado y aluminio, el uso de sellador de poliuretano para vidrios (ver figura 7).

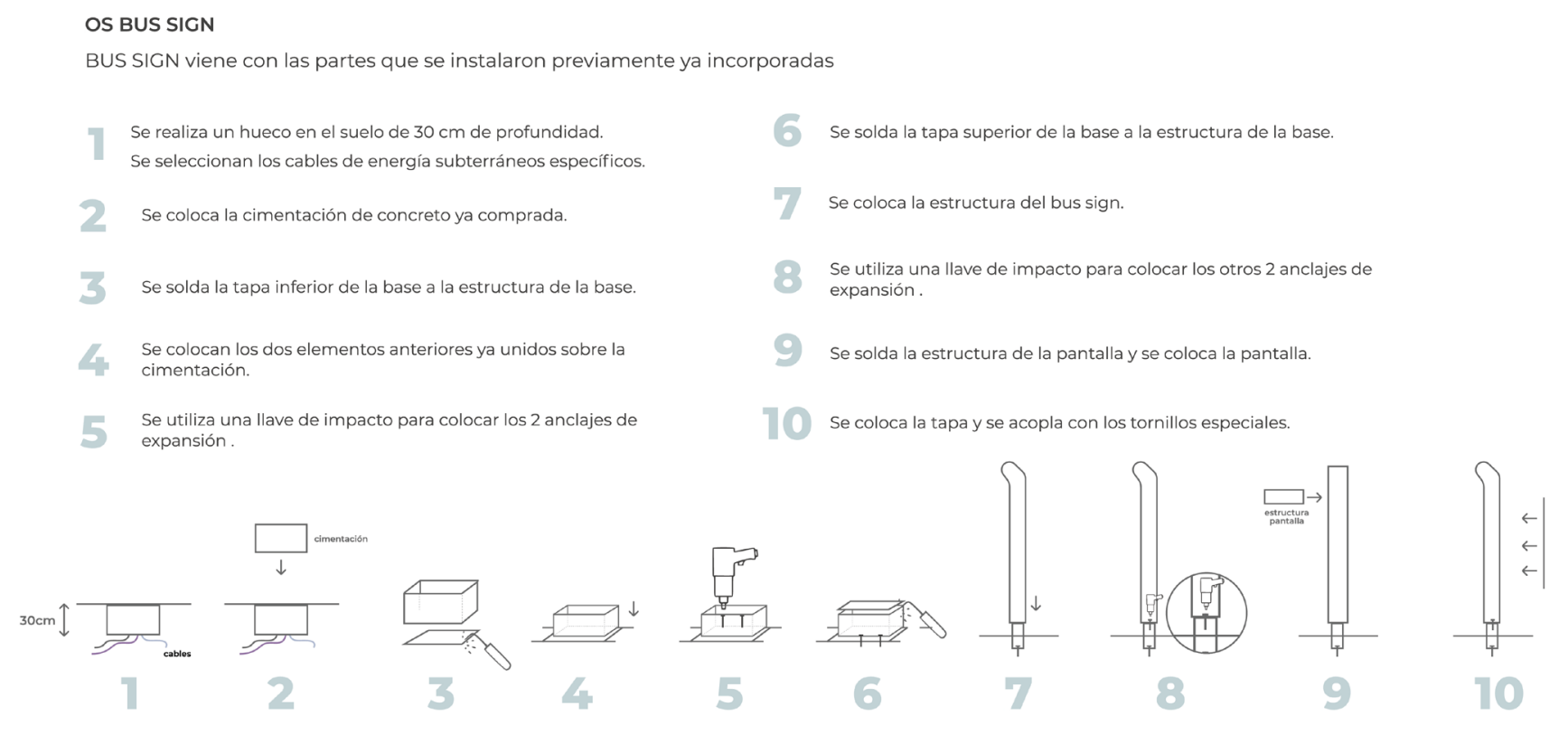

Figura 7. Manual de instalación OS bus sign

Fuente: Elaboración propia, 2020.

Por último, en la etapa de la simulación de diseño, se observó integralmente la síntesis de todas las etapas anteriores, con el objetivo de transmitir el proceso y el valor de la propuesta de diseño, por medio de un vídeo, en el cual se observaron las mejoras que se darían en el servicio con el uso del sistema.

\section{Discusión de resultados}

Cuando se compara el resultado obtenido, mediante el diseño de los objetos inteligentes, con lo que existe actualmente para solventar las necesidades expuestas, que presentan las paradas de buses convencionales, se puede notar que el sistema OS, del mismo modo que soluciones ya existentes, cuenta con iluminación y cámaras, sin embargo, lo que lo hace destacar a nivel de seguridad es la búsqueda de varias soluciones dentro del sistema para llegar a un resultado integral. Entre estas soluciones, se incorpora un botón de emergencia en caso de situaciones de riesgo, además, de acuerdo con estudios de inseguridad en el transporte público [11], se menciona que la incertidumbre es el primer factor que incide en las prácticas de movilidad, ya

4 Pulverización electrostática: técnica para aplicación de productos químicos utilizada con el fin de lograr mayor cobertura y deposición del producto asperjado, disminuyendo la dosis requerida para el tratamiento y reduciendo la pérdida de producto químico arrojado al ambiente [10]. 
que la información sobre el servicio se constituye como un elemento clave en interacción con la percepción de inseguridad y las preocupaciones de las personas; es así como esta sensación de inseguridad en la calles, a causa de la falta de información, lleva a la intranquilidad e incertidumbre con su desenvolvimiento en el uso del transporte público.

Por ello, se incorpora un sistema de información que permite brindar datos confiables a los ciudadanos que lo requieran, a diferencia de los "mupis" que se encuentran actualmente en las calles, la interfaz que muestra el sistema OS cuenta con una pantalla dividida. La cual, al lado izquierdo, permite la visualización en tiempo real de la ubicación de los buses y, al derecho, muestra los buses que se encuentran a 500 metros de distancia, a los que se les puede solicitar que se detengan. Esta solicitud se realiza por medio de botones, con el fin de evitar que las personas tengan que acercarse a la calle y solicitarlo con la mano.

A diferencia de las paradas convencionales, la persona no tiene que mantenerse pendiente a la llegada del bus, pues el sistema OS le brinda la posibilidad de escanear un código con su dispositivo móvil (ya sea celular, tableta, etc.) que muestra la ubicación del autobús seleccionado. Por último, el desarrollo integral de la seguridad se da debido a que se considera tanto el trayecto hacia la parada del autobús como la espera de este, ya que, actualmente, estos dos entornos se toman como independientes, cuando en realidad se deberían buscar soluciones que involucren ambos.

Para llegar a implementar este sistema de objetos inteligentes, se considera necesario ampliar la investigación de la interacción intangible con la pantalla y tomar en cuenta qué tan viable es desarrollar una aplicación móvil. De igual forma, se debe indagar en la optimización de costos con el fin de que los objetos sean rentables para la ciudad, para esto se podría considerar un diseño modular que facilite la sustitución de las partes que puedan dañarse. Además, buscar que los proveedores de la materia prima y de los componentes del diseño sean empresas que cuenten con programas basados en la economía circular y comprometidas con el medio ambiente, lo que permitiría el reintegro de los componentes y su posible revalorización. Por último, se podría investigar cómo implementar el uso de energía renovable y así generar un menor impacto ambiental.

\section{Conclusiones}

El desarrollo del proyecto permitió realizar distintos análisis que llegaron a hallazgos significativos, iniciando por la búsqueda de una problemática real que se vive en las ciudades actualmente, como lo es la inseguridad ciudadana, que complica el tránsito de los ciudadanos a diario; si bien es imposible eliminar inmediatamente este problema, sí se puede mitigar por medio de soluciones de diseño que aporten a un ambiente seguro. 
Durante el proceso, fue de suma importancia considerar la relación o relevancia de la seguridad tanto durante el trayecto como en la espera del bus, esto debido a que fue uno de los datos más recurrentes en las encuestas y estudios realizados con posibles usuarios. A causa de esto, durante la etapa de definición de la forma que tendría la propuesta, se definió que esta constaría de dos objetos inteligentes, interrelacionados y enfocados en generar una experiencia más segura en las ciudades por medio de un diseño con funciones inteligentes.

Fue necesario definir cómo deberían percibirse los objetos de acuerdo con el concepto establecido, ya que, para este caso, los ciudadanos regularmente generan asociaciones entre espacios oscuros y la sensación de inseguridad; materiales, formas o colores con el sentimiento de seguridad y si estos son tomados en cuenta, probablemente la aceptación de los objetos inteligentes en su contexto será mayor.

Además, por medio del modelado en 3D, se genera la documentación técnica requerida para el correcto funcionamiento y la comprensión del diseño.

Si se deseara llevar a cabo el desarrollo del producto, se recomienda iniciar por una validación por medio de prototipado físico, con el fin de realizar más pruebas con usuarios e identificar posibles mejoras o deficiencias que pueda presentar el producto en su morfología y funcionamiento. De esta manera, poder integrar estas mejoras y más adelante realizar pruebas para verificar la manufactura y su viabilidad de producción.

\section{Referencias}

[1] Nación, "El reto del transporte público", nación.com, [En línea]. Disponible en: https:// www.nacion.com/opinion/foros/el-reto-del-transporte-publico/6Y5CEYARFJA2LF72HBK 50W7FHA/story/ [Accedido: 30-sept-2020]

[2] Multimedios, Estas son las paradas más peligrosas para tomar el bus en San José, [En línea]. Multimedios, Disponible en: https://www.multimedios.cr/en-alerta/video-estas-sonlas-paradas-mas-peligrosas-para-tomar-el-bus-en-san-jose [Accedido: 30-sept-2020]

[3] Madrigal. R, "Paradas de autobús en San José se instalan sin coordinación y provocan caos en la ciudad", crhoy, [En línea]. Disponible en: https://archivo.crhoy.com/paradasde-autobus-en-san-jose-se-instalan-sin-coordinacion-y-provocan-caos-en-la-ciudad/ nacionales/ [Accedido: 30-sept-2020]

[4] Tushar.J, "What are the problems people generally face while traveling in a government bus or waiting for the bus in bus stop that can be technologically solved?", Quora, [En línea]. Disponible en: https://www.quora.com/What-are-the-problems-people-generallyface-while-traveling-in-a-government-bus-or-waiting-for-the-bus-in-bus-stop-thatcan-be-technologically-solved [Accedido: 30-sept-2020]

[5] L.Araya-Rojas, "Diseño de Objetos Inteligentes", Academia, [En línea]. Disponible en: https://www.academia.edu/40788559/Disen_o_de_Objetos_Inteligentes [Accedido: 17-diciembre-2020] 


\section{revistaIDI+}

[6] ElMundo, "Mujeres utilizan más y durante más horas el transporte público", elmundo. cr, [En línea]. Disponible en: https://www.elmundo.cr/costa-rica/mujeres-utilizan-mas-ydurante-mas-horas-el-transporte-publico/ [Accedido: 30-sept-2020]

[7] L.Pereyra, A. Gutiérrez, \& M. Nerome, La inseguridad en el transporte público del Área Metropolitana de Buenos Aires. Experiencias y percepciones de mujeres y varones, Territorio, [En línea]. Disponible en: https://www.researchgate.net/ publication/326162976_La_inseguridad_en_el_transporte_publico_del_Area_ Metropolitana_de_Buenos_Aires_Experiencias_y_percepciones_de_mujeres_y_varones/ fulltext/5b3c1e8ba6fdcc8506eed0e6/La-inseguridad-en-el-transporte-publico-delArea-Metropolitana-de-Buenos-Aires-Experiencias-y-percepciones-de-mujeres-yvarones.pdf [Accedido: 30-sept-2020]

[8] Amelia Rueda, "6 de cada 10 ticos usan transporte público, 20 puntos porcentuales debajo de la media mundial",ameliarueda.com, [En línea]. Disponible en: https://www. ameliarueda.com/nota/6-cada-10-ticos-usa-transporte-publico-20-debajo-mediamundial [Accedido: 30-sept-2020]

[9] Léxico, "diligente", lexico.com, [En línea]. Disponible en: https://www.lexico.com/es/ definicion/diligente [Accedido: 30-nov-2020]

[10] Darío.I, "LA PULVERIZACiÓN ELECTROSTÁTICA DE AGROQUÍMICOS: TEORÍA, EVALUACIONES Y APLICACIONES EN EL SECTOR AGRÍCOLA", Universidad Nacional de Colombia, [En línea]. Disponible en: https://repositorio.unal.edu.co/bitstream/handle/ unal/11813/98525305.\%202008_Parte1.pdf?sequence=3\&isAllowed=y\#: :text=La\%20 pulverización\%20electrostática\%20es\%20una,la\%20dosis\%20requerida\%20para\%20 el[Accedido: 1-dic-2020]

[11] Pereyra, L. P., Gutiérrez, A., \& Nerome, M. M. , La inseguridad en el transporte público del Área Metropolitana de Buenos Aires. Experiencias y percepciones de mujeres y varones, Territorio, [En línea]. Disponible en: https://www.researchgate. net/publication/326162976_La_inseguridad_en_el_transporte_publico_del_Area_ Metropolitana_de_Buenos_Aires_Experiencias_y_percepciones_de_mujeres_y_varones/ fulltext/5b3c1e8ba6fdcc8506eed0e6/La-inseguridad-en-el-transporte-publico-delArea-Metropolitana-de-Buenos-Aires-Experiencias-y-percepciones-de-mujeres-yvarones.pdf [Accedido: 30-sept-2020] 